\title{
Learning about Hygiene and Antibiotic Resistance through Mobile Games
}

\author{
Evaluation of Learning Effectiveness
}

\author{
Andreea Molnar \\ Lancaster University \\ Lancaster, United Kingdom \\ andreea.molnar@lancaster.ac.uk
}

\author{
Patty Kostkova \\ University College London \\ London, United Kingdom \\ P.Kostkova@ucl.ac.uk
}

\begin{abstract}
Edugames4all MicrobeQuest! is a mobile game that aims to teach microbiology and create awareness about important healthcare issues among 9 to 12 years old. This article presents the game, discusses the game design and integration of the learning objectives into the game mechanics. A pilot study has been performed to assess the game effectiveness in teaching the learning objectives integrated into the game. The study showed that the game can teach the learning objectives, however, the knowledge difference has not been statistically significant across all three learning objectives.
\end{abstract}

\section{CCS CONCEPTS}

- Applied computing $\rightarrow$ Interactive learning environments; Computers in other domains;

\section{KEYWORDS}

antibiotic resistance; children; health; hygiene; learning; mobile apps; serious games

ACM Reference Format:

Andreea Molnar and Patty Kostkova. 2018. Learning about Hygiene and Antibiotic Resistance through Mobile Games: Evaluation of Learning Effectiveness. In DH'18: 2018 International Digital Health Conference, April 23-26, 2018, Lyon, France. ACM, New York, NY, USA, 5 pages. https://doi.org/10. $1145 / 3194658.3194682$

\section{INTRODUCTION}

Currently, there is an increased ownership of mobile devices among children [1]. They could be used to complement teaching in an enjoyable manner through games. This paper will present a mobile game, edugames4all MicrobeQuest! [20], aimed at improving children knowledge about healthcare issues such as hygiene, infection prevention and responsible antibiotic use. A review of serious games for health [4] aimed at games that address interventions associated with infections and infection prevention and control has shown that the research in this area is still emerging with most studies focusing on the inception and development stages. In this

Permission to make digital or hard copies of part or all of this work for personal or classroom use is granted without fee provided that copies are not made or distributed for profit or commercial advantage and that copies bear this notice and the full citation on the first page. Copyrights for third-party components of this work must be honored.

For all other uses, contact the owner/author(s)

DH'18, April 23-26, 2018, Lyon, France

(C) 2018 Copyright held by the owner/author(s).

ACM ISBN 978-1-4503-6493-5/18/04.

https://doi.org/10.1145/3194658.3194682 study, we want to address part of the evaluation gap and present an evaluation of existing games for health.

A previous version of this game exists as a desktop version and the desktop version has previously been evaluated [9]. The mobile version of the game had only been evaluated for usability [21]. There are several challenges that can occur in using mobile devices for learning including the possibility of cognitive overload [23]. Therefore, the evaluation of the desktop version does not necessarily guarantee the same results as the mobile version, an evaluation of the mobile version of the game also being necessary. This research will focus on exploring whether the mobile game version improves the player's learning outcome.

In order to address this aim, the paper is organised as follows. The next section presents existing research on educational mobile games. The following section describes the Edugames4all MicrobeQuest!, what learning objectives are covered and how they have been integrated into the game mechanics. This section is followed by the evaluation and the results. This research ends with a presentation of the study's conclusion and proposed future work.

\section{GAMES FOR HEALTH}

Among STEM educational games, games that teach about healthcare issue are the most popular [3]. It is probably not surprising as serious games were embraced early by the medical community and are aimed at improving directly or indirectly the physical, mental and well-being of individuals.

Mobile games have been used to facilitate healthy living, rehabilitation or awareness about healthcare issues, such as improving medication compliance for elders [6], as a means to research the nutritional choices [12], promoting physical activity [13] or as part of telerehabilitation programs [7]. Positive results have been reported in the literature for improved medication compliance (tested in an 18 weeks user study), an improvement which has been higher for participants that had a previous interest in games [6]. OrderUP! [10], a game that aims to improve understanding of healthy eating, has shown to lead to players showing early engagement changing their eating habits [10].

As opposed to the above games, our game focuses on creating awareness about hygiene and responsible antibiotic use among children. Hand hygiene significantly reduces illness related absences [22], whereas antibiotic resistance is an ongoing concern and responsible antibiotic use could help alleviate this issue [8]. With the aim of addressing these issues, the desktop version of the game has been adapted [9] for play on mobile devices as an app [19]. This would allow us to assess how effective the app is in delivering the 


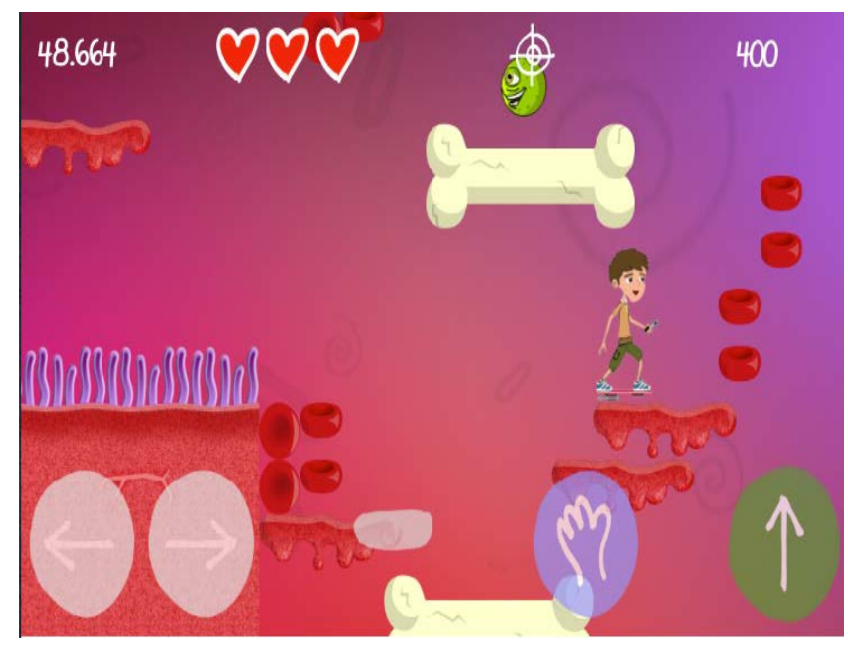

Figure 1: Player exploring the human body

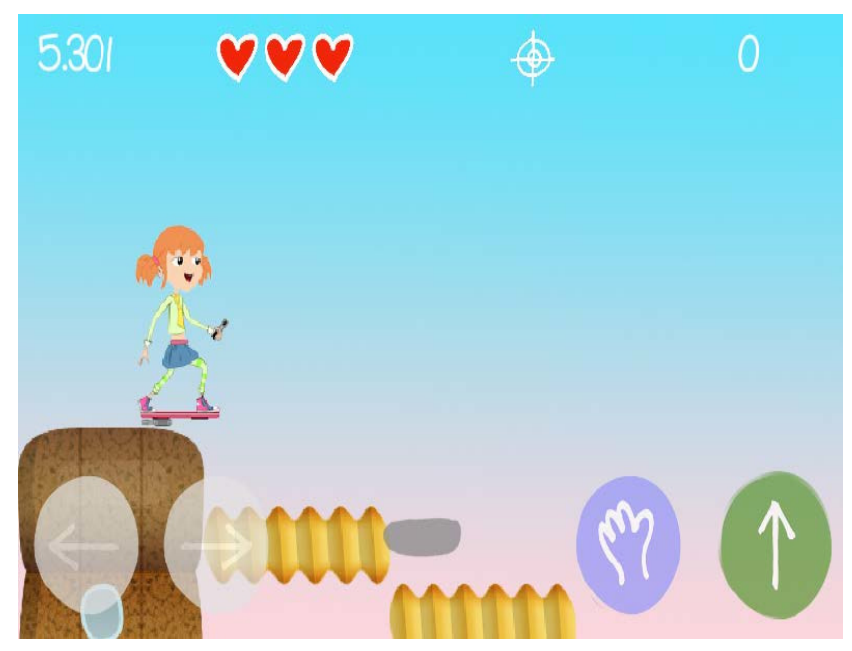

Figure 2: Player exploring the kitchen

educational message and the game's appeal for children in our age group.

A review of serious games for health [4] aimed at games that address interventions associated with infections and infection prevention and control has shown that the research in this area is still in emerging form with most studies focusing on the inception and development stages. Games that were evaluated and have been focused on educating children about antibiotic resistance [11], [18] have shown mixed results regarding their effectiveness. This research aims to address this gap and to focus on the effectiveness of the game on educating and creating awareness about hygiene and antibiotic awareness, as children are one of the population categories often ignored by the existing healthcare campaigns [16].

\section{EDUGAMES4ALL MICROBEQUEST!}

Edugames4all MicrobeQuest! is aimed at creating awareness about microbe transmission; food, body and hand hygiene; and responsible antibiotic use [20]. The game was designed to focus on learning objectives that would be covered in the primary school curriculum, however, some of its content could be useful for the general public.

The game is organised across different missions, starting with a tutorial mission aiming to familiarise the player with the game mechanics. This was motivated by two principles. First, there is empirical support showing that text based tutorials are not too effective [2,17], especially when working with children [17]. Second, having a tutorial level that slowly introduces the players to the game mechanics has been shown to be effective in teaching the game mechanics in other educational games aimed at children [17].

At the beginning of the game, the player is asked to select an avatar. The selected avatar then shrinks up to the size of a microorganism, allowing the player to interact with different microbes, bacterias and viruses (see Figure 1 and Figure 2). By shrinking the player, we can see the effect of different actions such as: what happens with the microorganism on the hand after washing them with soap, how the milk becomes yoghurt with the help of a bacteria, or what is the effect of not following the doctor's instructions when using antibiotics.

The game missions facilitate the exploration of different environments such as: human hand, human body (see Figure 1) or the food in the kitchen (see Figure 2). During these explorations, the player is taught the learning objectives through game mechanics and text incorporated into the game. For example, in order to teach the following learning objective: Our bodies have natural defences that protect us, through the game mechanics the player avatar, shrunken to the size of a microorganism and explores inside of the human body. Through the journey, the player encounters harmful microorganism. In order to protect the body and the player not to lose his game life s/he has to collect white blood cells and throw them at the harmful microorganism. The same learning objective would be reinforced through text, by displaying to the player: There are good and bad microbes. Good microbes can turn milk into yoghurt. That's how yoghurt gets made. Amazing isn't it?.

To facilitate the educational content assessment, the game allows for a similar integrated assessment of the educational content as the desktop version of the game [14]. The game allows assessing the player knowledge before and after being exposed to the learning objectives in games, though a quiz similar with "How to be a millionaire". This could be used to assess the effectiveness of the game without the need for additional questionnaires being delivered to the students outside the game but also to determine what aspects of the game are more effective in delivering the educational message and what others need to be improved. An evaluation of this kind of assessment on the desktop version of the game has shown that $63 \%$ of the players preferred it, as opposed to questionnaire before and after the game play [14]. Moreover, the quiz provides an opportunity to provide the players formative and summative assessment and feedback on their progress. 
Table 1: Statements of the Questions used to assess Player's Knowledge

\begin{tabular}{c|c} 
Q. No. & Question \\
\hline Q1 & If you cannot see a microbe it is not there \\
Q2 & Bacteria and viruses are the same \\
Q3 & Soap can be used to wash away bad bugs \\
Q4 & You should wash your hands after handling raw meat \\
Q5 & We use good microbes to make things like bread and yogurt \\
Q6 & Antibiotics can harm our good bacteria as well as bad bacteria \\
Q7 & Bacteria, viruses and fungi can be found in different enviroments \\
Q8 & Bacteria, viruses and fungi come in different shapes and sizes \\
Q9 & "Bad" microbes can make us ill \\
Q10 & Not all microbes are harmful \\
Q11 & Our bodies have defences to fight off disease \\
Q12 & Antibiotics can be used ot fight off bacterial infections \\
Q13 & If antibiotics are taken, it is important ot finish the course \\
Q14 & Vaccines can be used to obtain immunity to viral diseases \\
Q15 & Vacciness can be used to obtain immunity to viral diseases where the body's natural defences \\
Q16 & alone are not enough \\
& Bacteria are becoming resistant to many antibiotics due to antibiotic misuse (not finishing the course) \\
\hline
\end{tabular}

\section{EVALUATION}

The evaluation questionnaire was designed to cover the learning objectives presented in the section above. Learning objectives were assessed through a multiple choice questionnaire. These questions have also been used to assess the same learning objectives in other studies [9]. Table 1 presents the question's statements. The player had to state whether they agree with a given affirmation and had to choose between the following options: True, False and Don't know.

Paired sample t-test [5] was used to check whether there was a statistically significant difference between the players' answers to the questionnaire assessing their knowledge before the game (pre-test) and the questions assessing their knowledge after the playing session (post-test).

\subsection{Participants}

A total of 19 participants from different socio economic backgrounds and age groups took part in the study. The participants were recruited from schools in the UK and an after-school computer club. The children were recruited to match the age group the game was aimed for. Their average age was 9.2 years old, standard deviation 0.91 . Most of the participants were female $(n=11)$ and most of them were playing computer games on a regular basis.

\subsection{Set-Up and Methodology}

Before taking part in the study, consent was obtained from the school and after-school club, and from the parents of the children that took part in the study. All were provided with written information about the study. During the experimental section, the children were explained the study again (by the teacher and/or the researcher that carried out the study). They were given the opportunity to ask questions and were given the option to leave the study at any time (none of the participants opted for this and all of them fully participated in the study). Afterwards, they asked to complete a questionnaire collecting demographic information (age and gender) and assessing their previous knowledge of the learning objectives covered in the game. Next, the participants were given a mobile phone (Samsung Galaxy S4), that had the game already pre-installed. They were asked to play the game for thirty minutes. Afterwards, they were asked to fill a questionnaire that comprises of questions assessing their learning performance. The questions assessing the knowledge before playing the game were the same questions which assessed the knowledge after the game play.

\subsection{Results and Discussions}

To assess how much the players have learned we have compared the study participants responses to the pre-test questionnaire (administered before they played the game) to those of the post-test questionnaire (administered after the playing session). Figure 3 presents the player's answers to the pre-test knowledge questions. It presents how many players answers on average correct, incorrect or reporting that they do not know the answer to the given questions.

The Student t-test was used to determine whether there is a significant difference between the answer to the questions before and after playing the game, considering a $95 \%$ confidence interval. The results showed that for one of the learning objectives, $Q 8$ - Bacteria, viruses and fungi come in different shapes and sizes, the difference was statistically significant (see Table 1). For the other learning objectives, the difference between the pre and post test was not statistically significant. There are four reasons which might have contributed to these results. First, the results could be explained by the fact that the most of the players already know some of the concepts covered in the game (see Figure 3 for the participants answers to the questionnaire administered prior to the study). For example, all students knew the correct answer to the questions four before 


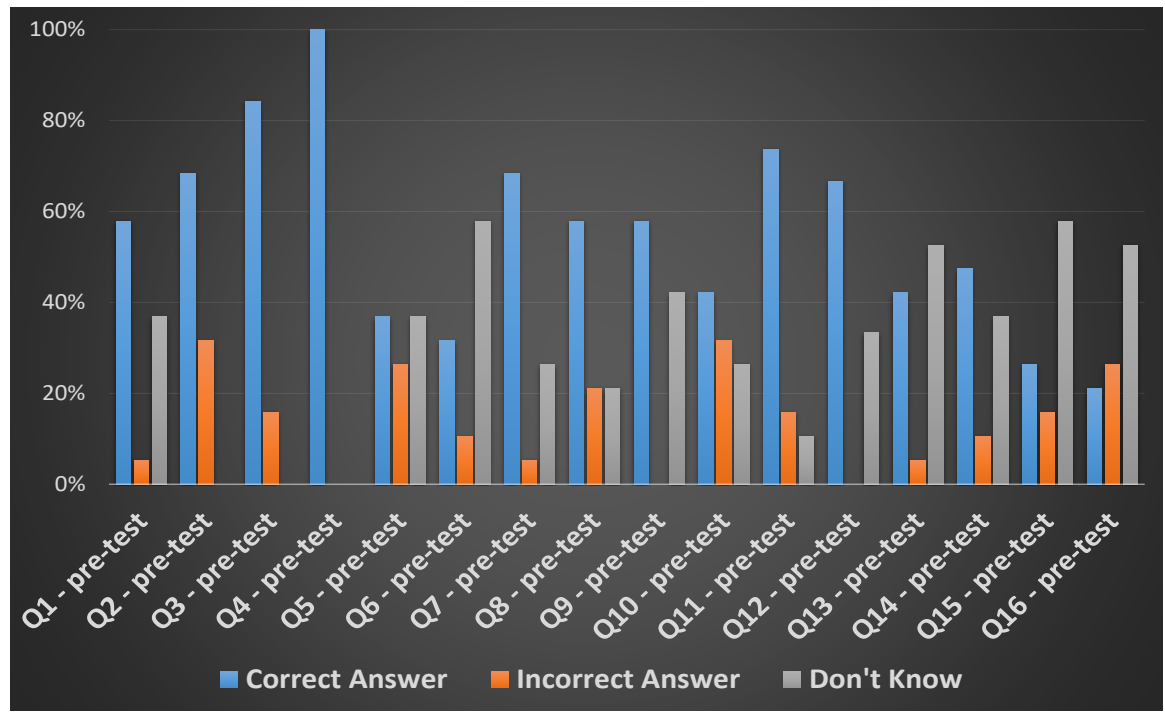

Figure 3: Players' answers to the pre-test questions

Table 2: Paired t-test Results

\begin{tabular}{c|c|c|c|c|c} 
Q. & Mean & $\begin{array}{c}\text { Std. } \\
\text { Deviation }\end{array}$ & $\begin{array}{c}\text { Std. Error } \\
\text { Mean }\end{array}$ & $\mathbf{t}$ & $\mathbf{p}$ \\
No. & & 0.99 & 0.24 & -0.48 & 0.63 \\
\hline Q1 & -0.11 & 0.72 & 0.17 & -0.32 & 0.74 \\
Q2 & -0.056 & 0.85 & 0.20 & 0.56 & 0.57 \\
Q3 & 0.11 & 0.11 & 1.00 & 0.33 \\
Q4 & 0.11 & 0.47 & 0.11 \\
Q5 & 0.05 & 0.99 & 0.23 & .23 & 0.81 \\
Q6 & 0.38 & 0.77 & 0.18 & 2.12 & 0.05 \\
Q7 & -0.11 & 0.75 & 0.17 & -.62 & 0.54 \\
Q8 & -0.44 & 0.85 & 0.20 & -2.20 & 0.04 \\
Q9 & -0.16 & 0.70 & 0.16 & -1.00 & 0.33 \\
Q10 & -0.38 & 1.09 & 0.25 & -1.51 & 0.14 \\
Q11 & -0.11 & 0.58 & 0.13 & -0.80 & 0.43 \\
Q12 & 0.17 & 0.63 & 0.15 & 1.14 & 0.26 \\
Q13 & -0.11 & 0.99 & 0.24 & -0.48 & 0.63 \\
Q14 & 0.35 & 1.11 & 0.27 & 1.30 & 0.21 \\
Q15 & 0.11 & 0.83 & 0.19 & 0.56 & 0.57 \\
Q16 & -0.05 & 0.93 & 0.22 & -0.25 & 0.80 \\
& & & & \\
\hline
\end{tabular}

playing the game: You should wash your hands after handling raw meat. Second, it might be due to the difficulty in executing some game mechanics in the game [20]. As a result, not all the students advance to the next level and were not exposed to all the learning objectives. Third, the traditional education is more effective for the children/young adults who do not usually play video games, and the gaming profile could affect the interest and motivation towards learning with a certain type of game [15]. Fourth, our sample was relatively small $(\mathrm{n}=19)$ and a larger sample size might have led to better results.

\section{CONCLUSIONS AND FUTURE WORK}

We presented edugames4all MicrobeQuest!, a mobile game aimed at teaching children microbiology. The game uses a combination of text and game mechanics to teach and reinforce the learning objectives. The learning objectives covered in the game are based on the primary school curriculum. This paper presented the results of a study assessing primary school children learning with the game. The results showed that the game could teach but the learning across all learning objectives was not statistically significant. As the sample size was small, we cannot confirm the hypothesis in one direction or another.

We are currently attempting to get a larger sample size and determine whether the difference in learning outcomes among different learning objectives still persist. We want to perform an experimental study assessing the differences in learning with mobile vs PC games both in formal and informal learning environments. We plan to take steps to increase the sense of immersion even further by complementing the teaching done through game mechanics not only through text but also through speech, as the children, especially those on the younger side found reading the text explanation tiring and often skipped over it. On a longer term, we want to assess whether the game leads to changes in children's attitude and behaviour towards hygiene and responsible antibiotic use. 


\section{ACKNOWLEDGMENTS}

Our thanks go to Fariz Benchaoui, Charlie Green and Jonny Wildey for developing the mobile app. Christina, Daly, Lucy Milner, Nikki Chester, Alison Minall and Nancy Clunie with helping with organising the study. The children who took part in the study and volunteered their feedback on the application. This research was partially supported by the University of Portsmouth Research Accelerator Fund and GADSA, Grant No: ES/P004733/1. The desktop version of the game was initially implemented as part of the e-bug, a European Commission funded project, DG SANCO, Grant no: 2005211.

\section{COMPETING INTERESTS}

The authors have declared that no competing interests exist.

\section{REFERENCES}

[1] 2014. One in Three Children has their own Tablet Computer. (2014). http: //media.ofcom.org.uk/news/2014/media-lit-audit-oct2014/ [Online; accessed 29-January-2015]

[2] Erik Andersen, Eleanor O’Rourke, Yun-En Liu, Rich Snider, Jeff Lowdermilk, David Truong, Seth Cooper, and Zoran Popovic. 2012. The impact of tutorials on games of varying complexity. In Proceedings of the SIGCHI Conference on Human Factors in Computing Systems. ACM, 59-68.

[3] Elizabeth A Boyle, Thomas Hainey, Thomas M Connolly, Grant Gray, Jeffrey Earp, Michela Ott, Theodore Lim, Manuel Ninaus, Claudia Ribeiro, and João Pereira 2016. An update to the systematic literature review of empirical evidence of the impacts and outcomes of computer games and serious games. Computers \& Education 94 (2016), 178-192.

[4] Enrique Castro-Sánchez, Yiannis Kyratsis, Michiyo Iwami, Timothy M Rawson, and Alison H Holmes. 2016. Serious electronic games as behavioural change interventions in healthcare-associated infections and infection prevention and control: a scoping review of the literature and future directions. Antimicrobial Resistance \& Infection Control 5, 1 (2016), 34

[5] Louis Cohen, Lawrence Manion, and Keith Morrison. 2013. Research methods in education. Routledge.

[6] Rodrigo De Oliveira, Mauro Cherubini, and Nuria Oliver. 2010. MoviPill: improving medication compliance for elders using a mobile persuasive social game. In Proceedings of the 12th ACM International Conference on Ubiquitous Computing. ACM, 251-260.

[7] Marcus Dithmer, Jack Ord Rasmussen, Erik Grönvall, Helle Spindler, John Hansen, Gitte Nielsen, Stine Bæk Sørensen, and Birthe Dinesen. 2016. The Heart Game: Using Gamification as Part of a Telerehabilitation Program for Heart Patients. Games for health journal 5, 1 (2016), 27-33.

[8] Julia Fahrenkamp-Uppenbrink. 2015. Countering antibiotic resistance. Science 347, 6226 (2015), 1109-1111.
[9] David Farrell, Patty Kostkova, Julius Weinberg, Lisa Lazareck, Dasun Weerasinghe, Donna M Lecky, and Cliodna AM McNulty. 2011. Computer games to teach hygiene: An evaluation of the e-Bug junior game. fournal of Antimicrobial Chemotherapy 66, suppl 5 (2011), v39-v44.

[10] Andrea Grimes, Vasudhara Kantroo, and Rebecca E Grinter. 2010. Let's play!: mobile health games for adults. In Proceedings of the 12th ACM international conference on Ubiquitous computing. ACM, 241-250.

[11] Alexander R Hale, Vicki Louise Young, Ann Grand, and Cliodna Ann Miriam McNulty. 2017. Can Gaming Increase Antibiotic Awareness in Children? A Mixed-Methods Approach. FMIR serious games 5, 1 (2017).

[12] Maria L Hwang and Lena Mamykina. 2017. Monster Appetite: Effects of Subversive Framing on Nutritional Choices in a Digital Game Environment. In Proceedings of the 2017 CHI Conference on Human Factors in Computing Systems. ACM, 4082-4096.

[13] Ruud H Knols, Tom Vanderhenst, Martin L Verra, and Eling D de Bruin. 2016. Exergames for Patients in Acute Care Settings: Systematic Review of the Reporting of Methodological Quality, FITT Components, and Program Intervention Details. Games for health journal 5, 3 (2016), 224-235.

[14] Patty Kostkova and Andreea Molnar. 2014. Educational Games for Creating Awareness about Health Issues: The Case of Educational Content Evaluation Integrated in the Game. In Medicine 2.0 Conference. JMIR Publications Inc., Toronto, Canada.

[15] Borja Manero, Javier Torrente, Clara Fernandez-Vara, and Baltasar FernandezManjon. 2017. Investigating the impact of gaming habits, gender, and age on the effectiveness of an educational video game: An exploratory study. IEEE Transactions on Learning Technologies (2017).

[16] Andreea Molnar. 2017. Children as agents of change in combatting antibiotic resistance. Journal of Health Services Research \& Policy (2017). https://doi.org/ 1355819617701512

[17] Andreea Molnar and Patty Kostkova. 2014. Gaming to master the game-game usability and game mechanics. In Serious Games and Applications for Health (SeGAH), 2014 IEEE 3rd International Conference on. IEEE, 1-7.

[18] Andreea Molnar and Patty Kostkova. 2015. Learning through interactive digital narratives. In Interactive digital narrative: history, theory and practice. Routledge, 200-210.

[19] Andreea Molnar and Patty Kostkova. 2015. Mind the Gap: From Desktop to App. In Proceedings of the 5th International Conference on Digital Health 2015. ACM, 15-16.

[20] Andreea Molnar and Patty Kostkova. 2016. Ubiquitous bugs and drugs education for children through mobile games. In Proceedings of the 6th International Conference on Digital Health Conference. ACM, 77-78.

[21] Andreea Molnar and Patty Kostkova. 2018. Teaching Hygiene and Responsible Antibiotic Use through a Mobile Game for Children. In Mobile Apps Engineering: Architecture, Design, Development and Testing.

[22] Inge Nandrup-Bus. 2009. Mandatory handwashing in elementary schools reduces absenteeism due to infectious illness among pupils: a pilot intervention study. American journal of infection control 37, 10 (2009), 820-826.

[23] Lung-Hsiang Wong and Chee-Kit Looi. 2011. What seams do we remove in mobile-assisted seamless learning? A critical review of the literature. Computers \& Education 57, 4 (2011), 2364-2381. 